\title{
Efficient Design of Flexible and Low Cost Paper-Based Inkjet-Printed Antenna
}

\author{
A. M. Mansour, ${ }^{1,2}$ N. Shehata, ${ }^{2,3,4}$ B. M. Hamza, ${ }^{1,2}$ and M. R. M. Rizk ${ }^{1,2}$ \\ ${ }^{1}$ Department of Electrical Engineering, Faculty of Engineering, Alexandria University, Alexandria 21544, Egypt \\ ${ }^{2}$ Center of Smart Nanotechnology and Photonics (CSNP), SmartCI Research Center of Excellence, Alexandria University, \\ Alexandria 21544, Egypt \\ ${ }^{3}$ Department of Engineering Mathematics and Physics, Faculty of Engineering, Alexandria University, Alexandria 21544, Egypt \\ ${ }^{4}$ Bradley Department of Electrical and Computer Engineering, Virginia Tech, Blacksburg, VA 24061, USA
}

Correspondence should be addressed to A. M. Mansour; amansour.ahmed@yahoo.com

Received 1 June 2015; Revised 8 September 2015; Accepted 9 September 2015

Academic Editor: Junping Geng

Copyright (C) 2015 A. M. Mansour et al. This is an open access article distributed under the Creative Commons Attribution License, which permits unrestricted use, distribution, and reproduction in any medium, provided the original work is properly cited.

\begin{abstract}
A new, efficient, flexible, and cheap antenna designed at $1.57 \mathrm{GHz}$ microstrip patch antenna based on simple inkjet printer with improved performance using silver nanoparticles ink is developed. The antenna is printed on a kind of flexible substrate "glossy paper," to offer the advantage of light and flexibility for different applications. The performance of silver nanoparticles ink has been studied through inkjet printing versus postsynthesis annealing and multilayer printing. The conductivity has been improved to have promising values up to $2 \Omega / \mathrm{cm}$ at temperatures up to $180^{\circ} \mathrm{C}$. The surface morphology of the circuits has been analyzed using SEM with mean diameter of the nanoparticles around $100 \mathrm{~nm}$, uniform surface distribution, and mean thickness of the printed layer around 230 microns. Also, a simple design of a coplanar waveguide (CPW) monopole Z-shaped antenna has been considered as an application of fabricated printed antenna using the studied silver nanoparticles ink through a cheap printer.
\end{abstract}

\section{Introduction}

Most of researchers try to design a low cost, simple, flexible, efficient, and compact size antenna with an environmental friendly process for most of our life applications in both commercial and military sectors. They are often incorporated into mobile radio communication devices, RFID tags, wireless sensors, and being mounted on the exterior of aircrafts and spacecrafts. In addition, it could be used in retail managements, items tracking, biomedical sensing, and power scavenging circuits [1-8]. Since there is a new trend of printing electronic circuits on flexible substrate, many printed circuits have been recently fabricated on a typical photo paper and PET substrate which are promising candidates for extremely low cost substrates for fabricating electronic circuits and RF structures $[9,10]$. Such substrates have excellent dielectric characteristics and low surface profile. Through the appropriate coating, they would be suitable for direct write methodologies such as conductive inkjet printing instead of the traditional metal etching techniques. In addition, paper and PET films are commercially available, cheap, and environmental friendly materials. Regarding the printing technique, the inkjet one is fast and simple and does not require a clean room for rapid and efficient printing electronics. Inkjet printing on flexible substrate is perfect as it jets the single ink droplet from the printer's nozzles to the desired position, so there is no waste unlike etching technique [10]. Using silver nanoparticles inks in inkjet-printing process ensure higher metal conductivity as it has been selected to implement the circuits and to improve the conductivity depending on their higher surface to volume ratio with a high accuracy and enhance thermal and electrical conductivity, compared to the bulk silver ink.

So, this work introduces a novel experimental study on the optimum conditions for using conductive silver nanoparticles in inkjet printing of flexible nanoantenna. The selected silver nanoparticles ink would be printed using a cheap printer compared to similar research work in recent 


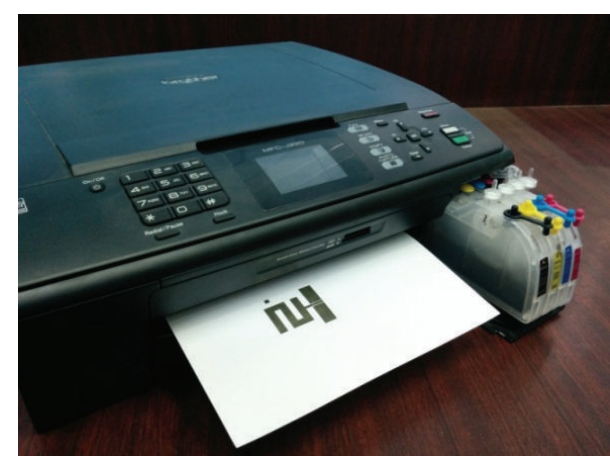

(a)

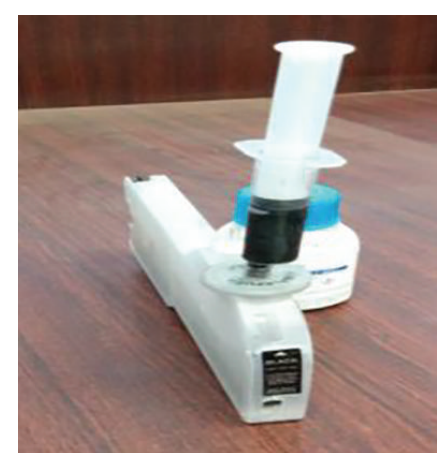

(b)

FIGURE 1: (a) Brother inkjet printer with the printed antenna. (b) Filling cartridge with conductive silver nanoparticles ink using syringe and filter.

literature and then analyzed its morphology and electrical conductivity at different postsynthesis heating temperatures and multilayer printing. Then, as an application to the analyzed sliver nanoparticles, a simple design of printed flexible antenna is presented with experimental and simulated analysis of the antenna performance. A simple coplanar waveguide (CPW) Z-shaped printed antenna, also known as microstrip antenna, is selected to be the printed antenna design due to its conformability and low cost as printed [11-15].

\section{Experimental Work}

The used silver nanoparticles ink is Mitsubishi NBSIJ-MU01, with viscosity of $2.30 \mathrm{mPa} \cdot \mathrm{s}$ and surface tension of $32 \mathrm{mN} / \mathrm{m}$. It is filled into a special cartridge to be fitted with the used Brother printer, as shown in Figure 1. This printer is extremely much cheaper compared to the famous printer Fujifilm Dimatix 2800 series which has been used recently in a lot of research papers in the printed electronics field. This printer cartridge is easy filling; we used a small plastic syringe to do this, with help of a disposable filter to keep any contaminations out of the print pipeline. The printing process is operated to generate one layer printed stripe and repeated to form multiple overlayered printed stripes.

The morphologies of the printed single-layered and multilayered printed antennas are analyzed using scanning electron microscope with JEOL GSM-6610LV, Japan, at an accelerated voltage up to $20 \mathrm{kV}$. In addition, the mean thickness of the printed single-layered antenna is obtained from SEM image for the cross section. In this paper, we use a simple design of printed antennas [12], with some changes in feeder spacing, and exposed printed antennas to different postsynthesis heating temperatures in a vacuum furnace at temperatures up to $180^{\circ} \mathrm{C}$ at different heating times. Then, the resistance is measured for the formed nanoprinted stripes with $1 \mathrm{~cm}$ distance between probes. The antenna is connected with a SMA connector using silver epoxy with ratio $1: 1$ and is dried at room temperature. The CPW fed monopole
Z-shaped antenna has been tested on Network Analyzer and the return loss is measured for the design. Also, we simulate Z-shaped antenna on the HFSS simulation tool and compare its results with the experimental one. More antenna characteristics have been experimentally analyzed such as VSWR and impedance. The geometry of the antenna is shown in Figure 2. The paper substrate has relative permittivity of 4.01 and loss tangent of 0.07 .

\section{Results and Discussions}

3.1. Printed Silver Nanoparticles Characterization. We start our research by finding the suitable and accurate conditions to achieve an optimum printing by testing the effect of temperature and multilayer printing. Table 1 shows the effect of expose samples to different temperature and how temperature could play an important role in fabrication process as we notice the resistance value decreases as temperature increases. The formed printed circuits are exposed to different temperatures and the resistance value reaches up to $2 \Omega / \mathrm{cm}$ at $180^{\circ} \mathrm{C}$, due to the increased mobility of the free electrons of the heated silver nanoparticles printed layer. The morphology and thickness measurement are shown in the SEM images of Figures 3 and 4 . Figure 3 shows the SEM image of the surface morphology of the silver nanoparticles ink printed on a glossy paper at room temperature, with smooth and uniform morphology. The mean diameter of the nanoparticles is found to be around $100 \mathrm{~nm}$, and the mean thickness of the printed layer around 230 microns with the probability of having minor cracks in the cross section but with homogeneous distribution of the nanoparticles, which could be compared to other similar research work with the ability of future extension to embed more conductive materials inside the ink $[16,17]$. Table 2 summarized the effect of multilayer printing with effect of temperature on the measured resistance. The overall resistance is higher than the resistance of singlelayered printed antenna. That could be explained due to the relative agglomeration of the printed silver nanoparticles while stacking in multilayers, in addition to the interfaces between the different layers, which could limit the mobility of free electrons of silver nanoparticles to move. 


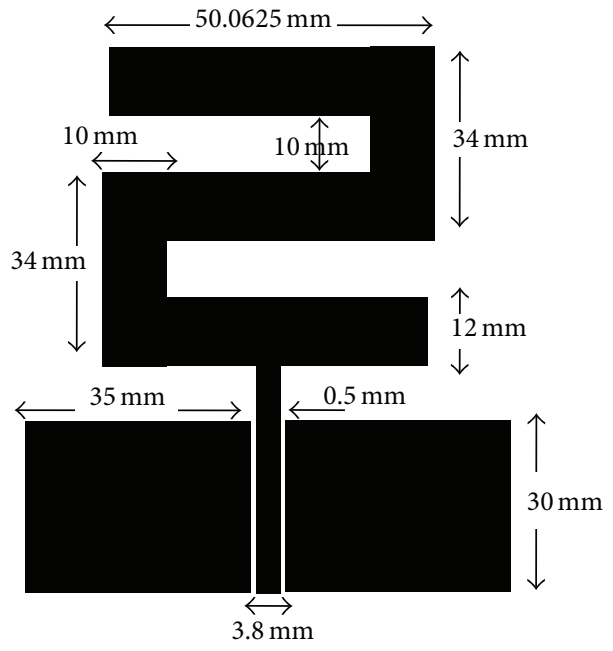

(a)

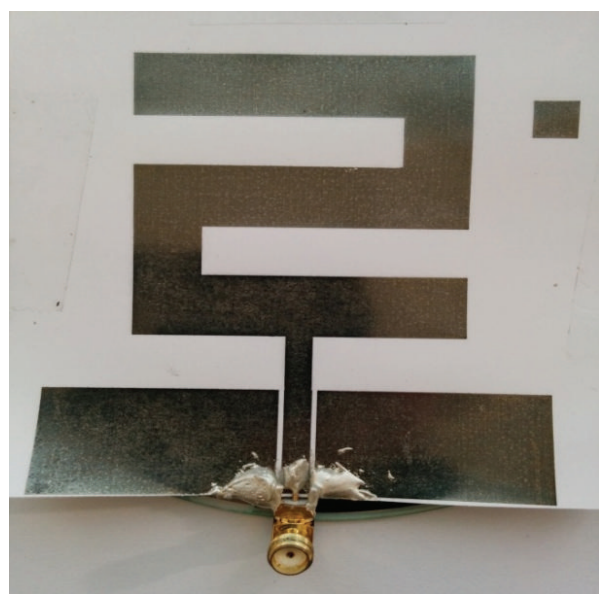

(b)

FIGURE 2: (a) Z-shaped CPW printed monopole antenna dimensions. (b) The printed version on glossy paper.

TABLE 1: Effect of temperature of one-layer sample on resistance in $\Omega / \mathrm{cm}$.

\begin{tabular}{|c|c|}
\hline Time (min) & Resistance $(\Omega)$ \\
\hline \multicolumn{2}{|c|}{ Temperature $=25^{\circ} \mathrm{C}$} \\
\hline 0 & $20 \mathrm{M}$ \\
\hline 5 & 7 \\
\hline 10 & 6 \\
\hline 15 & 4.5 \\
\hline 20 & 5.3 \\
\hline 25 & 5.1 \\
\hline \multicolumn{2}{|c|}{ Temperature $=55^{\circ} \mathrm{C}$} \\
\hline 0 & $20 \mathrm{M}$ \\
\hline 5 & 7.2 \\
\hline 10 & 5.3 \\
\hline 15 & 4.3 \\
\hline 20 & 5 \\
\hline 25 & 4.8 \\
\hline \multicolumn{2}{|c|}{ Temperature $=75^{\circ} \mathrm{C}$} \\
\hline 0 & $20 \mathrm{M}$ \\
\hline 5 & 4 \\
\hline 10 & 3.2 \\
\hline 15 & 3.3 \\
\hline 20 & 3.1 \\
\hline 25 & 2.7 \\
\hline \multicolumn{2}{|c|}{ Temperature $=180^{\circ} \mathrm{C}$} \\
\hline 0 & $20 \mathrm{M}$ \\
\hline 5 & 3.7 \\
\hline 10 & 3 \\
\hline 15 & 2.4 \\
\hline 20 & 2.1 \\
\hline 25 & 2.0 \\
\hline
\end{tabular}

TABLE 2: Effect of 2-layer and 3-layer printing under exposure to temperature on resistance in $\Omega / \mathrm{cm}$.

\begin{tabular}{|c|c|}
\hline Time (min) & Resistance $(\Omega)$ \\
\hline \multicolumn{2}{|c|}{ 2-layer, temp. $=25^{\circ} \mathrm{C}$} \\
\hline 0 & $20 \mathrm{M}$ \\
\hline 5 & 7 \\
\hline 10 & 5.4 \\
\hline 15 & 5.3 \\
\hline 20 & 4.6 \\
\hline 25 & 4.1 \\
\hline \multicolumn{2}{|c|}{ 2-layer, temp. $=75^{\circ} \mathrm{C}$} \\
\hline 0 & $20 \mathrm{M}$ \\
\hline 5 & $500 \mathrm{~K}$ \\
\hline 10 & $160 \mathrm{~K}$ \\
\hline 15 & 164 \\
\hline 20 & 150 \\
\hline 25 & 130 \\
\hline \multicolumn{2}{|c|}{ 3-layer, temp. $=25^{\circ} \mathrm{C}$} \\
\hline 0 & $20 \mathrm{M}$ \\
\hline 5 & 9 \\
\hline 10 & 8.8 \\
\hline 15 & 7.2 \\
\hline 20 & 5.8 \\
\hline 25 & 5.6 \\
\hline \multicolumn{2}{|c|}{ 3-layer, temp. $=75^{\circ} \mathrm{C}$} \\
\hline 0 & $20 \mathrm{M}$ \\
\hline 5 & $860 \mathrm{~K}$ \\
\hline 10 & $420 \mathrm{~K}$ \\
\hline 15 & 200 \\
\hline 20 & 192 \\
\hline 25 & 170 \\
\hline
\end{tabular}




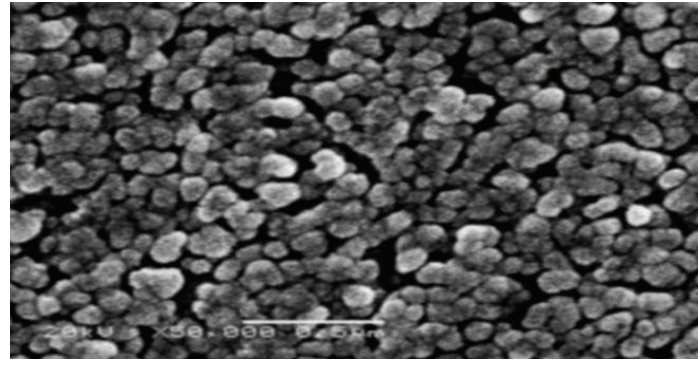

FIGURE 3: SEM image of the morphology of the one-layer printed silver nanoparticles ink on paper.

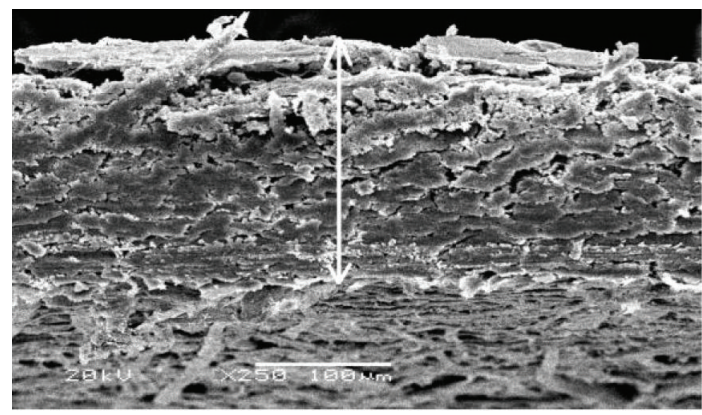

FIGURE 4: Cross-section image of the one-layer printed nanoantenna (the white line presents the printed silver nanoparticles ink and the glossy paper substrate is under the white line).

3.2. Printed Antenna Characterization. The experimental analysis of the return loss of the printed antenna from the Network Analyzer has been shown in Figure 5. In the same figure, it is compared to the simulation analysis which has been realized using Ansoft HFSS simulation tool for antenna design. The result as shown in Figure 5 of return loss $\left(\mathrm{S}_{11}\right)$ illustrates that the design of the Z-shape antenna has a notch at $1.57 \mathrm{GHz}$ at solution frequency equal to $1.4 \mathrm{GHz}$. Also, Figure 6 shows that VSWR is nearly $2 \mathrm{~dB}$ at the mentioned notch. This is promising to fabricate a flexible and cheap antenna with such values, which could be twisted and folded further in wide variety of applications, as the conductive silver nanoparticles contribute with the relative high surface/volume ratio and lower sheet resistance. The impedance of antenna is measured experimentally to have $12.5+j 75 \Omega$. Figures 7 and 8 show the radiation patterns of the gain of theta and phi at phi $=0$ and 90 degree, respectively. Choosing $1.4 \mathrm{GHz}$ as solution frequency leads to having more directive pattern of theta with high gain (around $-8 \mathrm{~dB}$ ).

\section{Conclusion}

This paper introduces a promising usage of silver nanoparticles in fabricating different antennas designs with a feasible and commercial method for printing using a commercial desktop printer. The printed silver nanoparticles show interesting conductivity values with postsynthesis heating temperatures. Also, we make a comparison between resistances' values when exposed to different temperatures for one/multi

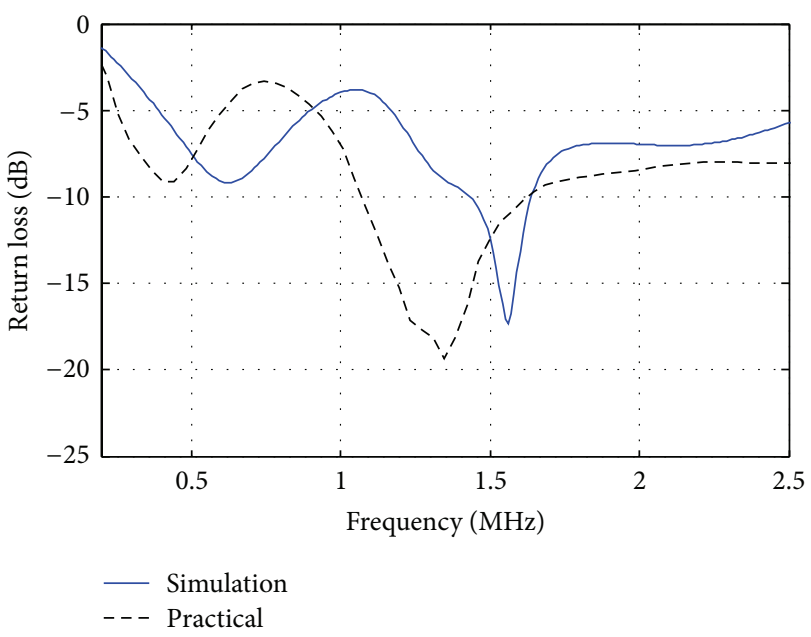

FIGURE 5: Return loss $\left(S_{11}\right)$ of the printed Z-shaped antenna on glossy paper.

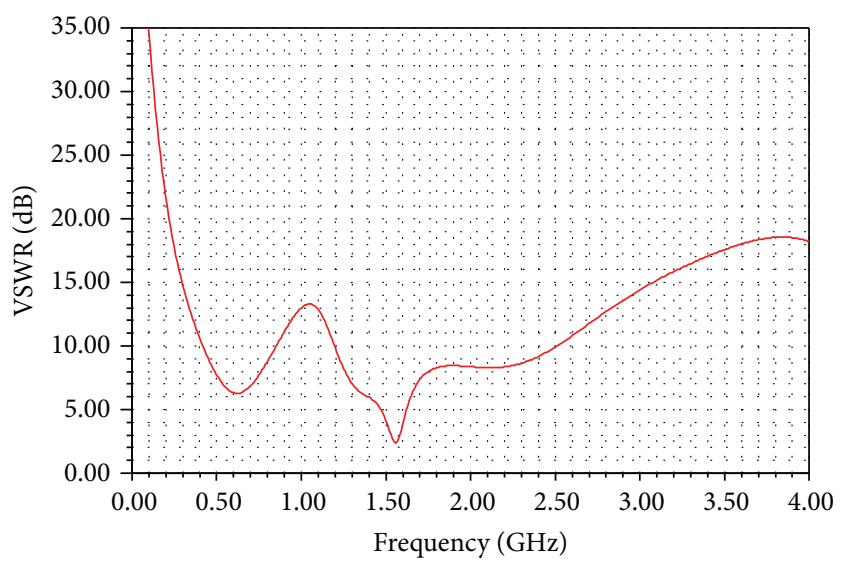

FIGURE 6: VSWR of the printed Z-shaped antenna on glossy paper.

(dB) GainTotal

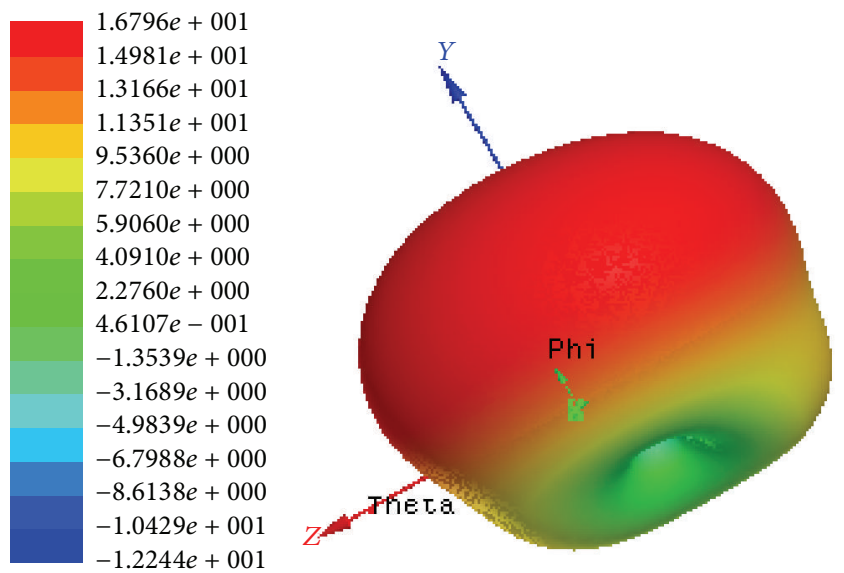

FIGURE 7: 3D model of the radiation pattern of the printed Z-shaped antenna. 


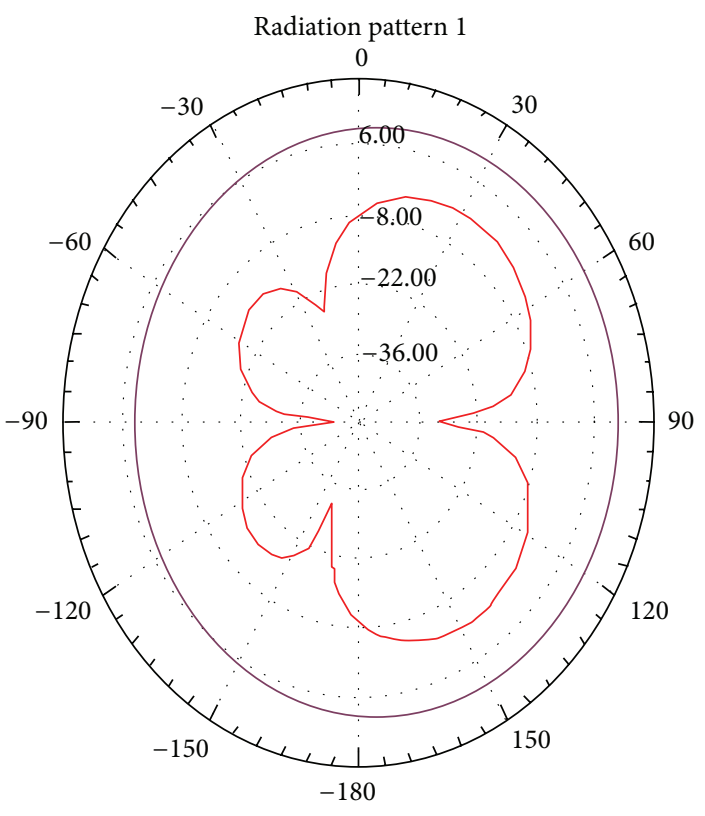

(a)

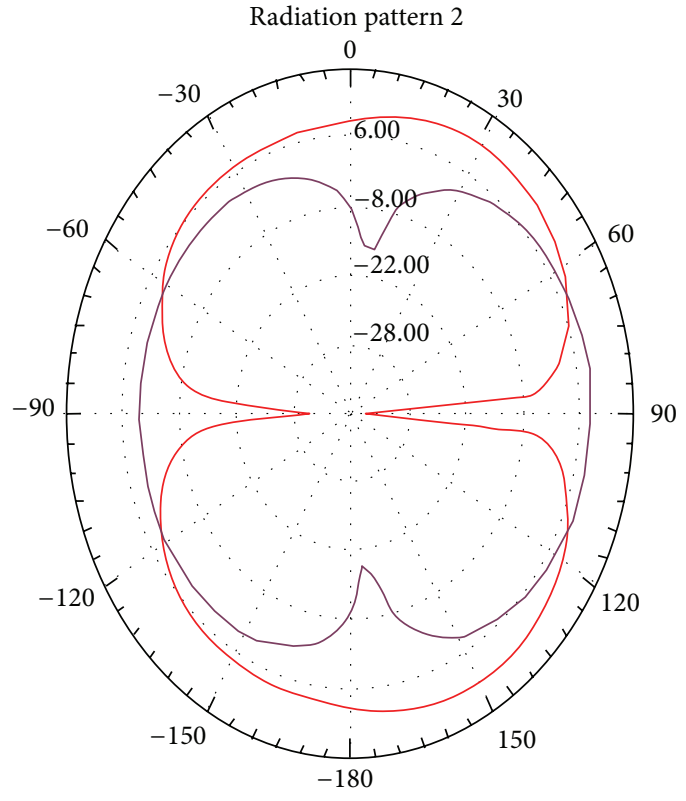

(b)

FIGURE 8: 2D model of the radiation pattern of the gain of theta and phi at phi $=0$ "to the left" and $90^{\circ}$ "to the right."

layers to clarify the impact of both post-treatment heating and the multi-printed layers. Moreover, a CPW monopole Zshaped antenna was realized via previous printing technique as an application of a simple printed antenna design of an RFID tag after heating treatment and selecting a suitable number of printing layers. The printed antenna using silver nanoparticles ink has been analyzed through different antenna characteristics including return loss, radiation pattern, VSWR, and impedance. This promising detailed study could lead to the development of flexible nanoantennas and generally flexible nanoelectronic circuits for wide variety of applications.

\section{Conflict of Interests}

The authors declare that there is no conflict of interests regarding the publication of this paper.

\section{Acknowledgments}

The authors appreciate the support of Dr. Mohammed Azab, Dr. Nazly Hassan, and Dr. Mohammed Al-Ansari in City of Scientific Research, Borg Al-Arab, Alexandria, for their help in postsynthesis heating and SEM imaging. Also, the authors would like to thank Dr. Ahmad Allam and Dr. Ahmad Shalaby from Egypt-Japan University of Science and Technology (E-JUST) for their technical support and experimental analysis of the printed antennas.

\section{References}

[1] K. Finkenzeller, RFID Handbook: Fundamental and Applications in Contactless Smart Cards and Identification, John Wiley \& Sons, New York, NY, USA, 2nd edition, 2003.
[2] K. V. S. Rao, P. V. Nikitin, and S. F. Lam, "Antenna design for UHF RFID tags: a review and a practical application," IEEE Transactions on Antennas and Propagation, vol. 53, no. 12, pp. 3870-3876, 2005.

[3] B.-K. Ang and B.-K. Chung, "A wideband E-shaped microstrip patch antenna for 5-6 GHZ wireless communications," Progress in Electromagnetics Research, vol. 75, pp. 397-407, 2007.

[4] R. Vyas, V. Lakafosis, A. Rida et al., "Paper-based RFID-enabled wireless platforms for sensing applications," IEEE Transactions on Microwave Theory and Techniques, vol. 57, no. 5, pp. 1370$1382,2009$.

[5] J. A. C. Theeuwes, H. J. Visser, M. C. van Beurden, and G. J. N. Doodeman, "Efficient, compact, wireless battery design," in Proceedings of the European Conference on Wireless Technology (ECWT '07), pp. 233-236, IEEE, Munich, Germany, October 2007.

[6] A. P. Sample, D. J. Yeager, P. S. Powledge, and J. R. Smith, "Design of a passively-powered, programmable sensing platform for uhf rfid systems," in Proceedings of the IEEE International Conference on RFID, pp. 149-156, Grapevine, Texas, USA, March 2007.

[7] J. A. Paradiso and T. Starner, "Energy scavenging for mobile and wireless electronics," IEEE Pervasive Computing, vol. 4, no. 1, pp. 18-27, 2005.

[8] J. P. Thomas, M. A. Qidwai, and J. C. Kellogg, "Energy scavenging for small-scale unmanned systems," Journal of Power Sources, vol. 159, no. 2, pp. 1494-1509, 2006.

[9] A. Rida, L. Yang, R. Vyas, S. Bhattaeharya, and M. M. Tentzeris, "Design and integration of inkjet-printed paper-based UHF components for RFID and ubiquitous sensing applications," in Proceedings of the 37th European Microwave Conference (EUMC '07), pp. 724-727, Munich, Germany, October 2007.

[10] L. Yang, A. Rida, R. Vyas, and M. M. Tentzeris, "RFID tag and RF structures on a paper substrate using inkjet-printing 
technology," IEEE Transactions on Microwave Theory and Techniques, vol. 55, no. 12, part 2, pp. 2894-2901, 2007.

[11] Y. Kawahara, S. Hodges, B. S. Cook, C. Zhang, and G. D. Abowd, "Instant inkjet circuits: lab-based inkjet printing to support rapid prototyping of UbiComp devices," in Proceedings of the ACM International Joint Conference on Pervasive and Ubiquitous Computing (UbiComp '13), pp. 363-372, Zurich, Switzerland, September 2013.

[12] Z. Konstas, A. Rida, R. Vyas, K. Katsibas, N. Uzunoglu, and M. M. Tentzeris, "A novel 'Green' inkjet-printed Z-shaped monopole antenna for RFID applications," in Proceedings of the 3rd European Conference on Antennas and Propagation (EuCAP '09), pp. 2340-2343, Georgia Institute of Technology, IEEE, Berlin, Germany, March 2009.

[13] R. Waterhouse, Printed Antennas for Wireless Communications, 2007.

[14] J. Sidén and H.-E. Nilsson, "RFID antennas-possibilities and limitations," in Radio Frequency Identification Fundamentals and Applications Design Methods and Solutions, C. Turcu, Ed., chapter 5, p. 324, InTech, Rijeka, Croatia, 2010.

[15] N. Sagars, "Wideband microstrip patch antenna using CPW feedline," in Proceedings of the International Academic Conference on Electrical, Electronics and Computer Engineering, Chennai, India, September 2013.

[16] K. Kumar and G. S. Kumar, "An experimental and theoretical investigation of surface roughness of poly-jet printed parts," Virtual and Physical Prototyping, vol. 10, no. 1, pp. 49-57, 2015.

[17] M. Vaezi, S. Chianrabutra, B. Mellor, and S. Yang, "Multiple material additive manufacturing - part 1: a review," Virtual and Physical Prototyping, vol. 8, no. 1, pp. 19-50, 2013. 

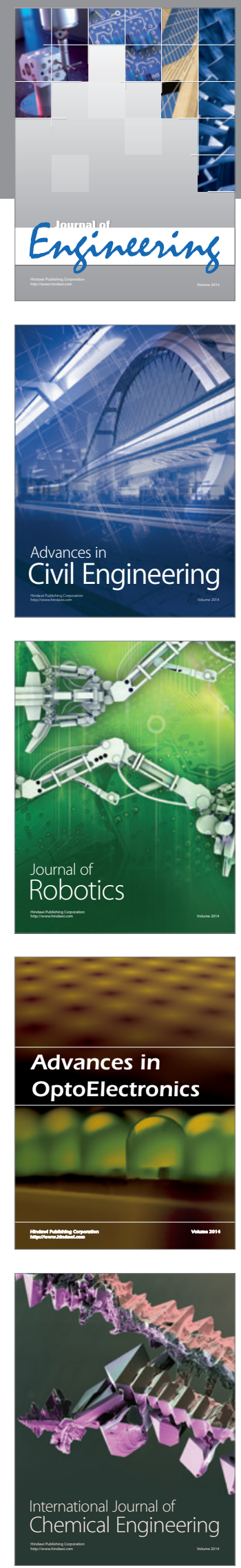

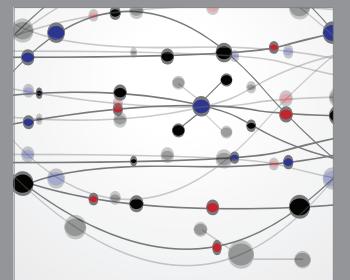

The Scientific World Journal
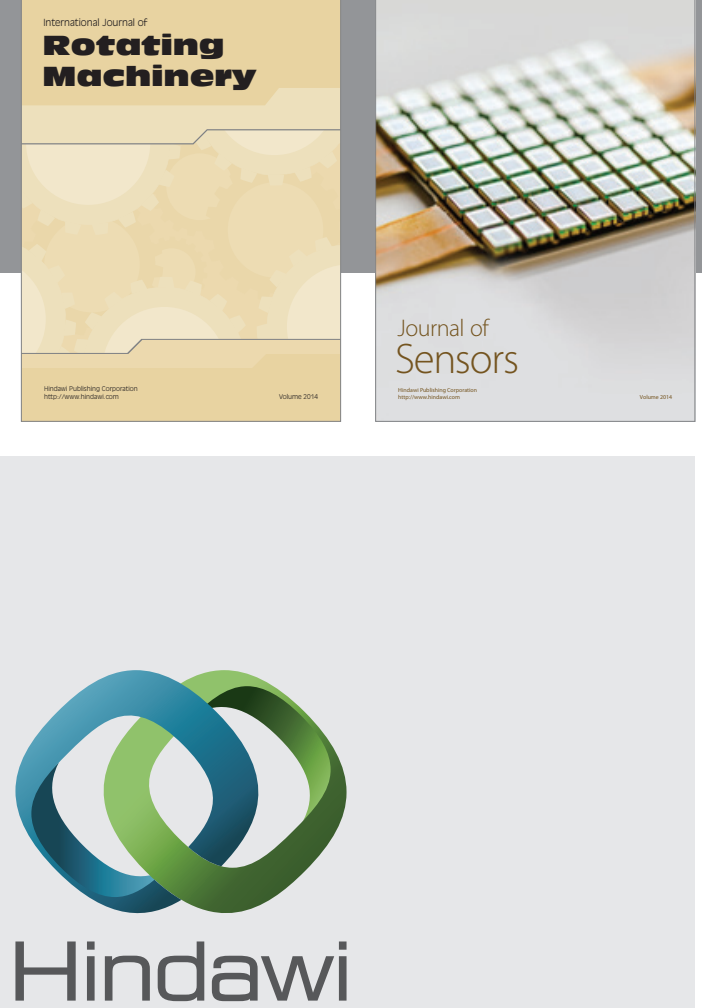

Submit your manuscripts at http://www.hindawi.com
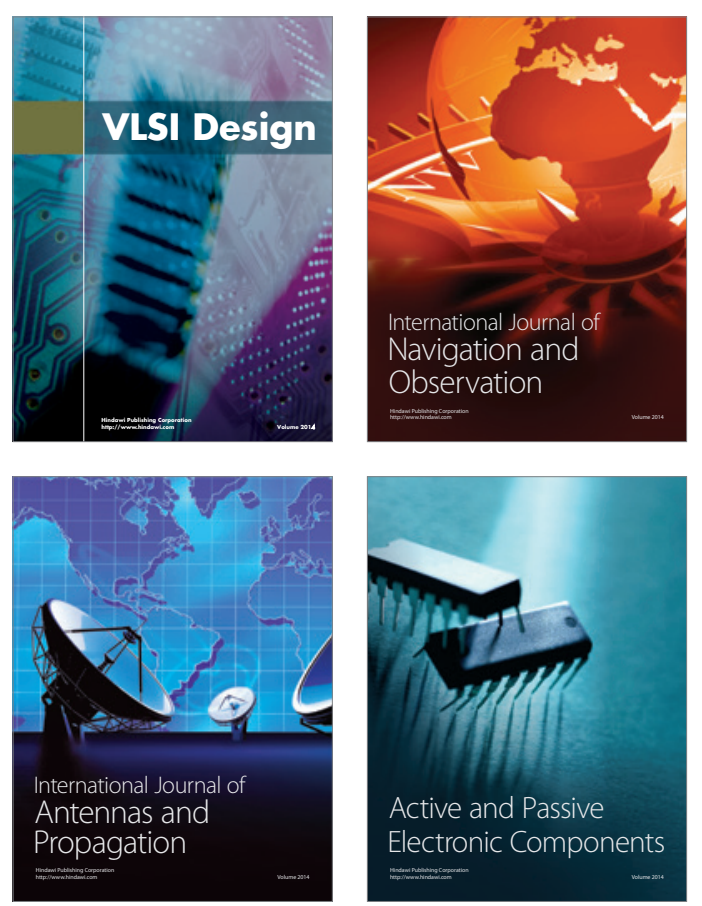
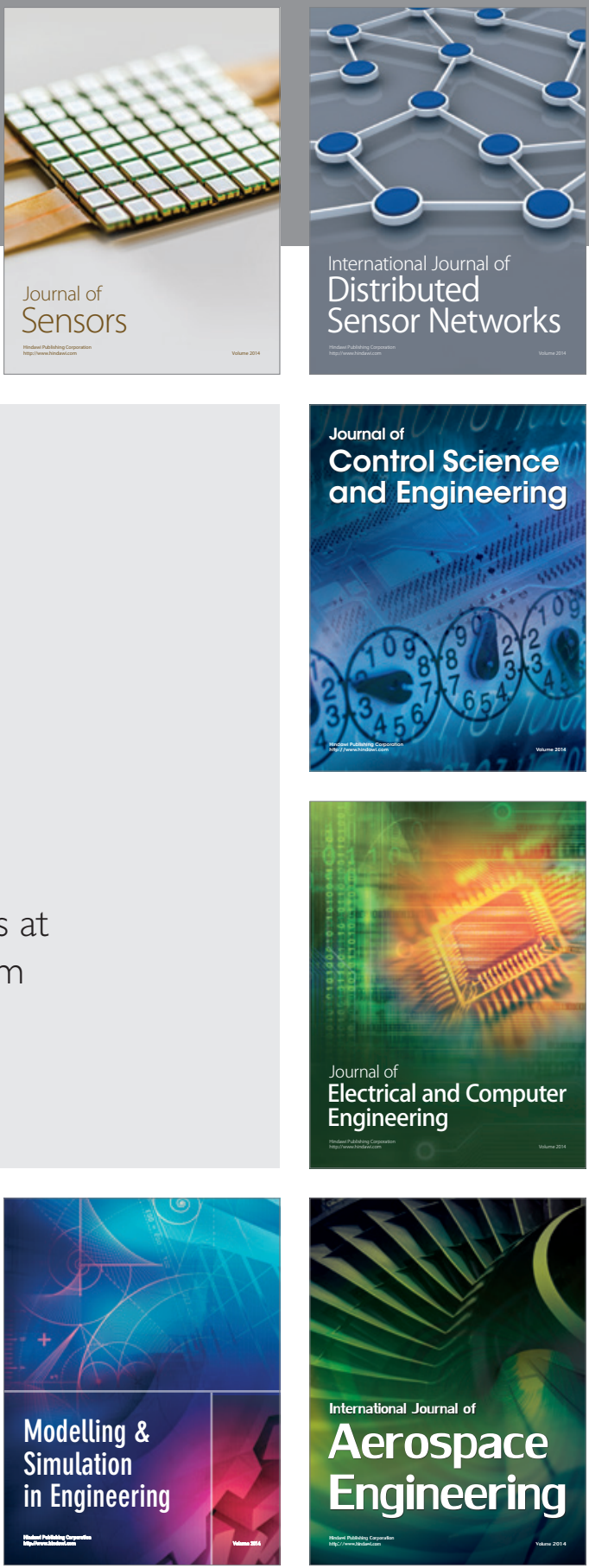

Journal of

Control Science

and Engineering
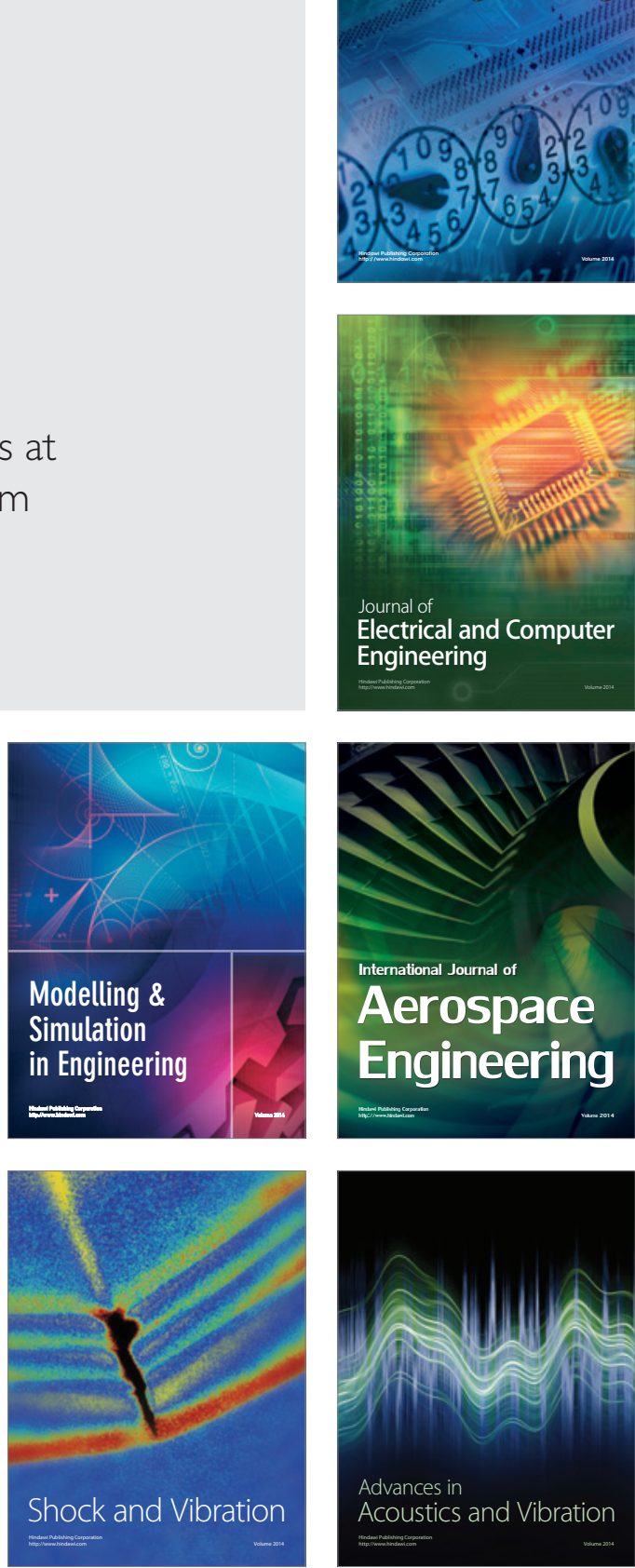